\title{
Sprachliches Wissen zwischen Lexikon und Grammatik
}

IDS-Jahrestagung, 9. bis 11. März 2010

Grammatiktheoretische Forschung, das hat die jüngste IDS-Jahrestagung wieder einmal plastisch vor Augen geführt, muss gedacht werden als zähes Ringen zweier grundsätzlich antagonistischer Prinzipien: Der reichhaltigen Fülle sprachlicher Okkurrenzen, deren gründlicher Auslotung ein beträchtlicher Teil der gegenwärtigen sprachtheoretisch und sprachtechnologisch ausgerichteten Anstrengung gewidmet ist, muss stets der Versuch gegenüberstehen, diese überbordende Varianz abstrahierend und generalisierend einzudämmen - ohne dabei die empirischen Befunde übermäßig und unzulässig zu nivellieren.

Die grundsätzlichen Kontroversen der aktuellen grammatikographischen Forschung standen im Fokus der diesjährigen IDS-Jahrestagung mit dem Titel „Sprachliches Wissen zwischen Lexikon und Grammatik“. Je nach theoretischer und empirischer Perspektivierung ist mit der Dichotomie „Grammatik und Lexikon“ zugleich auch das Verhältnis von Regularität und Idiosynkrasie in der Sprache angedeutet und damit die Konfrontation des Abstrakten (in Form von komplexen sprachlichen Einheiten, die der Sprecher aufgrund seines regulären Sprachwissens bilden könnte) mit dem Konkreten (realisiert in den Einheiten, die man zum korrekten Gebrauch und Verständnis schlicht kennen muss). Und damit ist gegenwärtig eben auch (wieder) die Grauzone dazwischen angesprochen, die mitunter so großangelegt eingeschätzt wird, dass sie die vorgenannten Prinzipien verdrängt und „das strikt Regelhafte und das rein Idiosynkratische“ schließlich „nur noch als Randbereiche des sprachlichen Wissens" erscheinen lässt, wie es Gert Webelhuth in seinem Auftaktvortrag formulierte. Unmittelbar zuvor allerdings hatte sich Stefan Engelberg, Leiter der Abteilung Lexik des IDS und des IDS-Projekts „Polysemie und konstruktionelle Varianz", im Wortsinn in medias res begeben: Das im Titel nur angedeutete „Zwischen“ kann ja auf verschiedene Art und Weise konzeptualisiert werden. Zum einen kann es einfach eine Grenze markieren; forschungsrelevant ist dann einzig die Frage, wo genau diese Grenze verläuft. Sprachtheoretische Ansätze, die reich gestaltete Lexikoneinträge postulieren und einen sehr schlank gehaltenen grammatisch-syntaktischen Apparat darüber operieren lassen, konkurrieren dabei mit solchen, die das Lexikon klein halten, indem sie die Einträge so minimal wie möglich spezifizieren, was natürlich ausgleichend potente grammatisch-syntaktische Regeln fordert. Verhandelt wird dabei also der jeweilige Anteil von Grammatik und Lexikon in einem arbeitsteiligen Prozess. Im Gegenzug kann aber jenes „Zwischen“ auch als eine Art hypostasiertes „Dazwischen“ angesehen werden, welches von den umgebenden Bereichen des ,reinen Lexikons“ oder der ,,reinen Grammatik" prinzipiell nicht abgedeckt wird. Und je nachdem, wie umfassend dieser Zwischenraum modelliert wird, kann er sogar auf den Gesamtbereich sprachlicher Erscheinungen ausgedehnt werden. Lexikon, Grammatik und alles dazwischen verschmelzen in diesem Konzept zu einem einzigen „Konstruktikon", dessen konkrete Inhalte in Form von komplexen Konstruktionen sich mal mehr, mal weniger regelhaft verhalten. Und so wurde 
dann im Verlauf der Tagung in verschiedenen Vorträgen an der offenbar recht wackligen Grenze zwischen Grammatik und Lexikon gerüttelt. Allen voran tat dies der Syntaktiker Gert Webelhuth (Frankfurt a.M.) im Eröffnungsvortrag ${ }^{1}$ mit dem Titel „Paradigmenwechsel rückwärts: Die Renaissance der grammatischen Konstruktion“. Webelhuth skizzierte die verschiedenen grammatischen Eigenschaften von Relativsätzen im Deutschen und präsentierte unterschiedliche Möglichkeiten grammatischer Analysen, etwa die, das jeweilige Verb als Kopf der Relativsatzkonstruktionen zu deklarieren. Dann müsste man aber für jedes dieser Verben sechs homophone Lexikoneinträge postulieren. Wenn man andererseits leere Elemente zu Köpfen mache, lässt das die Gemeinsamkeiten der sechs Relativsatzkonstruktionen wie Zufälle aussehen. Der einzige Grund für derartige Analysen, vermutete Webelhuth, liege ohnehin im Versuch, Konstruktionen um jeden Preis zu vermeiden. Seiner Meinung nach könne aber ein intelligent konzipiertes Vererbungsnetzwerk besser mit solchen Phänomen umgehen; konstruktionsbasierte Grammatiken seien somit nichts anderes als eine Rückkehr zur traditionellen Grammatik mit weiterentwickelten theoretischen Werkzeugen, nachdem sich Grammatiktheorien ohne Konstruktionen als empirische und theoretische Fehlentwicklungen erwiesen hätten. Wolfgang Imo (Münster) führte dann den sich in den folgenden Tagen als zentral erweisenden Terminus der Granularität ein. Unter dem Titel „Where does the mountain stop? Konstruktionen, Zeichen und Granularität" illustrierte Imo das Granularitäts-Konzept zunächst metaphorisch an der Grenzziehung zwischen ineinander übergehenden Bergen im Himalaja genaue Grenzen lassen sich ausschließlich im Rahmen von konkreten Untersuchungen mit jeweils genau bestimmten Untersuchungszielen bestimmen. Auf die Sprache übertrug Imo das anhand einer umfassenden Typologie des deiktischen Elements jetzt aus gesprächsanalytischer Sicht. Dieses jetzt stellt lediglich auf der gröbsten Betrachtungsebene eine „Augenblicksmarke " dar, bei feinerer Körnung werden zusätzliche Facetten wie „temporale $^{\text {c }}$ und ,gesprächsstrukturierende Augenblicksmarke " notwendig. Fraglich ist letztendlich aber, ob eine Grammatiktheorie überhaupt mit unterschiedlichen Korngrößen umgehen könne. Bei beliebig verfeinerter Detailschärfe kommt eine Vielzahl von Funktionen zum Vorschein, die im Extremfall bis hin zur Aufhebung der ja eigentlich in der grammatischen Beschreibung angestrebten Dekontextualisierung reicht. Diese Betrachtungsweise tendiere letztlich dazu, Konstruktionen zum Verschwinden zu bringen; es gibt dann am Ende nur noch holistisch zu beschreibende Einzelvorkommen. Den Granularitätsbegriff schließlich griff dann beispielsweise später Joachim Jacobs (Wuppertal) indirekt wieder auf. Anhand einer minutiösen Betrachtung der nur bei oberflächlicher Betrachtung distinkt erscheinenden Kategorien „Wort" und „Syntagma“" zeichnete Jacobs die notorisch schwierige Abgrenzung der drei linguistischen Teildisziplinen Morphologie, Syntax und Phraseologie nach und illustrierte damit jenen eingangs angedeuteten grundsätzlichen Antagonismus zwischen Generalisierung und Grenzziehung. Die einschlägigen empirischen Daten liefern unzählige Grenzfälle und seien letztlich so differenziert, dass die beiden Großkategorien „Wort" und „Syntagma“ (so attraktiv sie sowohl für theoretische Modelle als auch in der linguistischen Praxis sein mögen) bei Anlegung strenger wissenschaftlicher Standards nicht zu halten sind. Das verdeutlichte Jacobs zunächst an Beispielen aus dem Bereich der Wortbildung. So sprechen etwa bei Komposita einige Kriterien für eine Zuordnung zu Wörtern, andere für eine Zuordnung zu Syntagmen. Die strenge Distinktion der beiden Kategorien ist damit in Frage gestellt, die übliche Lösung sei die Abspaltung

Die weiteren Zusammenfassungen bilden nicht mehr die chronologische Reihenfolge der Vorträge ab. 
einzelner Stufen von Kriterien. So gelten dann viele Komposita zwar als Wörter, allerdings nicht als phonologische Wörter, da sie sich in phonologischer Hinsicht eher wie Phrasen verhalten. Anhand von zahlreichen graphematischen, morphologischen und syntaktischen Besonderheiten anderer lexikalischer Einheiten verdeutlichte Jacobs, dass sich für jede neue Stufung des Kriteriensystems weitere Gruppen von Problemfällen einfinden; es müssen stets neue Stufen separiert werden - bis hin zu Extremfällen, in denen jede Stufe nur noch eine einzige Eigenschaft voraussagt. Die Problematik ähnelt erkennbar der von Imo geschilderten, die Schlussfolgerung lautete allerdings anders: Die Eigenschaften von sprachlichen Ausdrücken können auch bestimmt werden, ohne die Eigenschaftsdimensionen zu Stufen (wie ,syntaktisches Wort", ,graphematisches Wort" oder schlechthin „Wort") zusammenzufassen. Prinzipiell denkbar jedenfalls, so das Fazit von Jacobs, wäre eine „Grammatik ohne Wörter" (so der Vortragstitel) allemal. In einer solchen, so die abschließend von Jacobs formulierte Hoffnung, könnte sogar der Blick frei werden für die tatsächlichen Zusammenhänge. Das wiederum erinnerte sehr an den Appell des Korpuslinguisten Patrick Hanks (Prag): Anstatt über Grenzen zu streiten, sollten Linguisten lieber Prototypen identifizieren und untersuchen. Daraus werde sich in vielen Fällen von selbst ergeben, was womit korreliert. Unter dem Titel „Wie man aus Wörtern Bedeutungen macht: Semantische Typen treffen syntaktische Dependenzen" entwickelte Hanks seinen Ansatz einer von Grund auf korpusgetriebenen, rein induktiven Sprachbeschreibung, bei der den intrinsischen und invarianten Eigenschaften lexikalischer Elemente, denen jeweils durch den Kontext semantische Rollen zugewiesen werden, bestimmte Implikaturen zugeordnet sind. Das heißt: Jedes Muster ist mit einer Bedeutung verbunden, zu beschreiben als Reihe von prototypischen Annahmen. Bemerkenswert erscheint einerseits die damit verbundene Technik der Errechnung wahrscheinlicher Bedeutungen eines Verbs in einem konkreten Kontext, andererseits aber auch die Möglichkeit, sowohl konventionelle Sprachgebrauchsmuster zu integrieren als auch mit randständigeren „Sprachgebrauchsmuster-Exploitationen“ adäquat umzugehen, ohne die linguistisch angestrebte Charakterisierung von Sprachen als Systemen mit regelgeleitetem Verhalten in Frage stellen zu müssen. Diese Regeln differenzierte Hanks damit in ein Set von Regeln, das den Normalgebrauch von Wörtern reguliert, und ein weiteres interagierendes Set, das die Abweichungen von der Norm reguliert. Somit können also auch ,irreguläre" Kontexte auf regelgeleitete Weise Bedeutung determinieren.

Konträr zu diesem Versuch, den Regelbegriff zu retten - wobei hier Regeln natürlich nur als statistische Wahrscheinlichkeiten anzusehen sind und nicht als absolute Notwendigkeiten - unterbreitete Anatol Stefanowitsch (Bremen) den wohl weitreichendsten Vorschlag zur Abschaffung der Regeln. Seinen Standpunkt, dass es „Keine Grammatik ohne Konstruktionen" (so der Titel) geben könne, vertrat Stefanowitsch mit der Argumentation, es müsse ja ein konstruktionsverarbeitendes kognitives System geben, da viele komplexe Einheiten der Sprache nicht (ausschließlich) regelbasiert produziert und verstanden werden können. Ein solches System sei auch in der Lage, kompositionelle Strukturen verarbeiten zu können - ein rein regelverarbeitendes System dagegen hat mit idiomatischen Strukturen naturgemäß Probleme und bedarf noch einer anders gearteten Ergänzung. Wenn man also die unbefriedigende Variante zu vermeiden sucht, dass Grammatiken mit zwei grundsätzlich verschiedenen Systemen arbeiten, sei ein konstruktionsbasiertes Modell besser geeignet, grammatische Strukturen einheitlich zu repräsentieren. Das verdeutlichte Stefanowitsch unter anderem an der Familie der intensivierenden W-Konstruk- 
tionen à la „Was in aller Welt soll das alles?" oder „Warum in aller Welt sollte man ein Rockkonzert besuchen?" Ähnliche Überlegungen (mit allerdings durchaus anderen Ergebnissen) klangen auch im Vortrag von Edeltraud Winkler, Kristel Proost und Stefan Engelberg (beteiligt, aber nicht vortragend Svenja König; alle IDS) an, der eine Art Werkstattbericht aus dem derzeit am IDS laufenden Forschungsprojekt „Polysemie und konstruktionelle Varianz" darstellte. Hauptziel des Projekts ist die Erstellung eines Handbuchs zur Beschreibung korpusbasiert ermittelter Argumentstrukturmuster einerseits und der darin partizipierenden Verben andererseits. Während der Arbeiten zu diesem Handbuch wurde allerdings schnell klar, dass die Argumentstrukturmuster nicht eindeutig zu identifizieren sind und die Fülle an Idiosynkrasien deskriptiv kaum zu erfassen ist. Untersuchungen an verschiedenen Argumentstrukturmustern des Deutschen (der Such-Konstruktion mit Beispielen der Art „die Innenstadt nach Ladendieben durchkämmen"; den Geräusch-als-Bewegungsverben, etwa: „Die Harleys knattern über die Landstraßen “ und Resultativkonstruktionen wie „Er redet sich in Rage ${ }^{c}$ ) deuten darauf hin, dass Varianz empirisch tatsächlich weniger in Form einer polysemen Strukturierung ,großer“ Konstruktionen als vielmehr in Form von Familienähnlichkeiten zwischen „kleinen“, granulareren Konstruktionen vorkommt. Das heißt, Varianz drückt sich durch formale und semantische Beziehungen zwischen einzelnen Argumentstrukturmustern in einem Netz von Familienähnlichkeiten aus. Konkret kann daraus gefolgert werden, dass verbspezifische Füllungspräferenzen für Argumente in bestimmten Argumentstrukturmustern und Partizipationsbeschränkungen für Verben hinsichtlich der Muster nach valenznahen lexikalischen Beschreibungen verlangen. Insbesondere argumentspezifische Beschränkungen für bestimmte Argumentstrukturmuster (wie z.B. das erwähnte „sich in Rage reden") verlangen nach der lexikalischen Beschreibung argumentstrukturmusterspezifischer Kollokationen. Weitere Kandidaten für sprachliche Phänomene zwischen Lexikon und Grammatik präsentierte Tibor Kiss (Bochum) unter dem Titel: „Distribution und Interpretation von Präposition-Substantiv-Kombinationen. "Solche Kombinationen, die aus einer Präposition und einem artikellosen zählbaren Substantiv im Singular bestehen, etwa: „unter Voraussetzung (des Einverständnisses)“, scheinen nicht vollkommen frei kombinierbar. Tatsächlich scheint es insgesamt zwei reguläre Varianten zu geben, nämlich eine semantisch endozentrische und kompositionell aufgebaute, sowie eine nicht-endozentrische und eher nicht kompositionelle (etwa: „auf Seite seiner Verteidiger ${ }^{c}$ ), die demnach als Konstruktion zu bezeichnen wäre. Den einschlägigen Problemen näherte sich Christiane D. Fellbaum (Princeton) von eher technischer Seite in ihrem Vortrag über die "Abgleichung von WordNets Verbinventar mit Argumentalternationen in FrameNet". Die beiden im Titel genannten großen lexikalischen Ressourcen sind ursprünglich mit der Intention, menschliches Sprachwissen für die konkrete maschinelle Anwendung bereitzustellen, konstruiert worden. Während WordNet als semantisches Netz (und damit praktisch als Modell des mentalen Lexikons) konzipiert ist, stellt FrameNet die Verben primär framesemantisch dar und bezieht somit auch die syntaktischen Realisierungsmuster mit ein. Beide Ressourcen erstellen aber Form-Bedeutungspaare mit bestimmten Inkompatibilitäten, was nicht der Fall sein sollte, wenn die These Bestand hat, dass gemeinsame Argumentstrukturalternationen semantisch homogene Verbklassen anzeigen. Der Versuch, die beiden Ressourcen zu harmonisieren, dürfte somit auch der sprachwissenschaftlichen Theoriebildung förderlich sein. Annelies Häcki Buhofer (Basel) berichtete unter dem Titel „Lexikographie der Kollokationen zwischen Anforderungen der Theorie und der Praxis" zum einen über die Entwicklung eines Kollokationenwörterbuchs mit dem 
Titel „Wörterbuch der festen Wendungen und typischen Wortverbindungen des Deutschen $^{\text {". }}$ Zum anderen schilderte sie die Hindernisse der Integration von korpusbasiert ermittelten Kollokationen ins „Neue Baseldeutsch-Wörterbuch“. Für beide Projekte jedenfalls hielt sie es im Fazit für geboten, reine Korpusanalysen mit Methoden der traditionellen Wörterbucharbeit zu kombinieren. Daran anknüpfen konnte Ulrich Heid (Stuttgart) in seinem Vortrag über die „Korpusbasierte Beschreibung der regionalen Variation von Kollokationen: Deutschland - Österreich - Schweiz - Südtirol“". Im Vordergrund standen dabei die Schwierigkeiten des Versuchs, in Kollokationsdaten aus Korpora Anhaltspunkte für regionale Variation zu finden - inbesondere unter der Maßgabe, lexikalisch eher unauffällige Kollokationskandidaten finden zu wollen. Das heißt: Nicht der "Zustupf", den der Schweizer leistet, ist hier im offensichtlichen Gegensatz zum "Zuschuss", den der Deutsche gibt, interessant - sondern eben die Kollokatorenwahl. Das Schweizerische „leistet ${ }^{\text {" }}$ eben in diesem Fall, das Deutsche, gibt". Trotz aller geschilderten Schwierigkeiten konnte Heid über ein Verfahren berichten, dessen angestrebtes Ziel darin besteht, Kollokationskandidaten in Korpora zu finden und im gleichen Schritt deren detaillierte Beschreibung (etwa nach Wortklassen und morphosyntaktischen Merkmalen) zu liefern. Beatrice Primus (Köln) beschäftigte sich unter dem Titel „Semantische Rollen zwischen Verbspezifizität und Generalisierung" mit der Frage, ob das unpersönliche Passiv im Deutschen am adäquatesten lexikonbasiert, beschränkungsbasiert oder konstruktionsgrammatisch zu beschreiben sei. Eine Auswertung von Experimentaldaten, Korpusbelegen und Erkenntnissen aus sprachvergleichenden Untersuchungen brachten Primus $\mathrm{zu}$ dem Schluss, dass es beim unpersönlichen Passiv zwischen verbspezifischen Rollen (etwa: Lesender/Gelesenes bei lesen) und verallgemeinerten strukturellen Rollen („ProtoRollen" wie Agens und Patiens) grammatisch relevante Involviertheits-Rollen gibt, deren Partizipanten auf einer Agens-Prototypikalitätsskala verortet werden können, wobei der sprachvergleichend invariant attestierte Bedeutungskern jeweils die merkmalreichste und logisch stärkste Agens-Lesart ist. Intra- und intersprachliche Variation und nachlassende Grammatikalität gibt es dann erst bei den merkmalsärmeren Agens-Lesarten. Erklärungen, die auf metaphorische Übertragung, konstruktionsgrammatische Vererbung oder Schemabildung zurückgreifen, wären damit überflüssig. Rückendeckung bekam Primus dabei aus den Reihen der Hirnforschung. Ina Bornkessel-Schlesewsky (Marburg/Leipzig) und Matthias Schlesewsky (Mainz) zeigten aus neurophysiologischer Perspektive, dass das menschliche Sprachverstehenssystem mit der Interpretation verbfinaler Strukturen beginnt, bevor das Verb vernommen wird; unbelebte Actor-Argumente beispielsweise bewirken messbare elektrophysiologische Reaktionen. Die im Vortrag mit dem Titel „Dynamische Aspekte der Argumentinterpretation: Eine sprachübergreifende Perspektive“ präsentierten Messungen von ereigniskorrelierten Potenzialen (also spontaner elektrischer Aktivität des Gehirns bei der Präsentation verschiedener sprachlicher Stimuli) lieferten allerhand Evidenz für die These, dass aufgrund von prototypischen Handlungsrelationen zwischen Actor und Undergoer (,Argument-Argument-Dependenzen ") thematische Hierarchien aufgebaut werden, die eine verbunabhängige Interpretation in Echtzeit ermöglichen. Claudia Maienborn (Tübingen) beschäftigte sich mit dem „Strukturausbau am Rande der Wörter" und unterzog adverbiale Modifikatoren beim Zustandspassiv (das sie nicht eigentlich als Passiv, sondern als Spezialfall einer deverbalen Kopula-AdjektivKonstruktion analysierte) einer genaueren Betrachtung. Maienborn zeigte, dass die lexikalische Adjektivierung Auswirkungen auf die gesamte Verbalphrase hat und die adverbialen Modifikatoren dabei vollständig in den Verbalkomplex integriert werden. Damit liegt 
diese Konstruktion (verdeutlicht am Beispiel „Das Manuskript ist von Chomsky zitiert“) also zwischen phrasalen Einheiten wie dem Vorgangspassiv („Das Manuskript ist von Chomsky zitiert worden") und Wortbildungen mit adjektivischen Köpfen („Das Manuskript ist Chomsky-zitiert"). Als Phänomen der semantischen Unterbestimmtheit stelle diese Struktur ein der Grammatik frei verfügbares Ausdrucksmittel (im Grenzbereich zwischen Wort und Phrase) für die Konzeptualisierung von Ad-hoc-Eigenschaften an der Grammatik-Pragmatik-Schnittstelle dar. Vieles sei damit gar nicht so idiosynkratisch, wie auf den ersten Blick anzunehmen. Die Annahme von Konstruktionen wäre damit nicht unbedingt notwendig. Eine Schlussfolgerung, die dann später auch Gereon Müller (Leipzig) dezidiert ausformulierte - das von ihm geforderte Arbeitsprogramm „Stück für Stück weg mit den Konstruktionen" markierte den Schluss seines Vortrags. Auf dem Weg dahin unterzog Müller unter anderem die „verblosen Direktiva“ (etwa: „In den Müll mit diesen Klamotten!'c) einer Reanalyse. Diese werden in neueren Publikationen häufig als Einheiten gehandelt, deren Analyse regelbasiert sehr schwierig, konstruktionsbasiert aber plausibel zu machen ist. Müller analysierte solche Äußerungen im Rahmen des minimalistischen Regelsystems als Verb-Erst-Sätze mit hinzugefügtem Antipassiv-Morphem, in deren Kopf allerdings ein Verb steht, dessen phonologische Realisierung null ist. So lieferte Müller also Analysen des Typs, wie er schon ganz zu Beginn von Webelhuth attackiert worden war, womit die theoretische Debatte also eigentlich wieder bei ihrem Ausgangspunkt angekommen wäre.

Gemäß dem sogenannten „Lernbarkeitskriterium “ sollte sich eine Sprachtheorie aber auch immer praktisch am Spracherwerb bewähren. Wie die theoretischen Grundannahmen des konstruktionsgrammatischen Paradigmas anhand von Studien zum Erwerb des Deutschen umgesetzt und getestet werden können, illustrierte Heike Behrens (Basel) in ihrem Vortrag über „Die Grenzen des lexikalischen Lernens: Konstruktionsprozesse im Spracher-

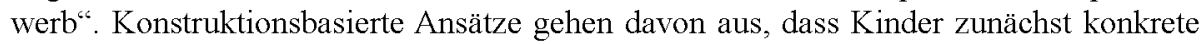
komplexe Sprachvorkommen beherrschen lernen, aus denen sie dann erst später abstrakte Konstruktionen generalisieren. Diese liegen dann wiederum der Fähigkeit zugrunde, neue Äußerungen kreativ zu generieren. Behrens wies mittels verschiedener praktischer Experimente, empirischer Daten und statistischer Methoden nach, dass Kinder dabei nicht etwa aus einem unbegrenzten Hypothesenraum schöpfen, sondern als „vorsichtige Generalisierer $^{c i}$ wahrscheinliche Strukturen auf der Basis des jeweiligen Sprachstandes generieren. Ziel der konstruktionsgrammatisch geleiteten Spracherwerbsforschung müsse es demnach sein, die Granularität der Generalisierung und die Interaktion der Verarbeitungsfaktoren $\mathrm{zu}$ ermitteln. Eine „Modularisierung“, davon zeigte sich Behrens überzeugt, stellt sich jedenfalls immer als Resultat der sprachspezifischen Ausdifferenzierung im Verlauf der Entwicklung dar - und nicht als der Beginn des Sprachlernens. Die Herausforderung für eine Linguistik des 21. Jahrhunderts müsse es deshalb sein, die spezifische Interaktion von linguistischen und nichtlinguistischen Variablen in einem sich entwickelnden Organismus angemessen zu modellieren. Auch Rosemarie Tracy (Mannheim) konnte in ihrem Vortrag über „Konstruktion und Rekonstruktion: Evidenz aus der Spracherwerbs- und der Sprachkontaktforschung" verdeutlichen, dass eine Mischung aus genetischer Ausstattung, sprachlichem Angebot der Umwelt und kognitiven Prinzipien (wie Mustererkennung, Intentionszuschreibung und dergleichen) notwendig ist, um den Spracherwerb von Kindern adäquat beschreiben und erklären zu können. An zahlreichen empirischen Belegen kindlicher Sprachäußerungen, insbesondere im Zusammenhang mit der Ausbildung von 
Verbklammerphänomenen, demonstrierte Tracy, wie die anfängliche Koexistenz heterogener Formate im Laufe der Entwicklung auf spezifische abstrakte Strukturen hin konvergiert. Das heißt, zunächst holistisch gegebene Formeln werden unter dem Druck verschiedener gleichzeitig vorliegender Strukturoptionen reanalysiert und restrukturiert. Zugleich aber wies Tracy darauf hin, dass Lerner bekanntlich früh Intuitionen über Wohlgeformtheit sowie Paraphrasenbeziehungen, Äquivalenzen etc. entwickeln. Das bereitet auch gängigen Konstruktionsgrammatiken Probleme. Es stellt sich also weiterhin die Frage: Wie bilden wir dieses Wissen ab?

Das erkennbar vielfältige Tagungsprogramm wurde noch durch diverse Rahmenereignisse flankiert, die hier nicht unerwähnt bleiben sollen. Parallel zu den Vorträgen fand eine Messe zur elektronischen Lexikographie statt, auf der 16 nationale und internationale Projekte ihre Forschungsergebnisse präsentierten. Außerdem gab es im Anschluss an die Tagung erstmalig einen korpuslinguistischen Workshop, der Linguistinnen und Linguisten die Grundlagen der korpusgestützten Datenerhebung vermittelte. Eine besondere Form der Anerkennung schließlich wurde dem Institut für Deutsche Sprache als Institution zuteil: Der im Rahmen der Jahrestagung traditionell verliehene Konrad-Duden-Preis der Stadt Mannheim ging dieses Mal an das IDS selbst. Damit würdigte die Jury die Gesamtleistung einer Forschungseinrichtung, welche in diesem Fall „die Vielfalt sprachwissenschaftlicher Erforschung der deutschen Gegenwartssprache repräsentiert". Das fügt sich sicherlich schön ein in den Rahmen einer Tagung, die das Spektrum der Zugänge und Möglichkeiten grammatischer Theoriebildung eindrucksvoll zu umfassen versuchte. Der erweiternde Anschluss ist dann auf der nächsten IDS-Jahrestagung zu erwarten, wenn vom 15. bis 17 . März 2011 unter dem Titel „Deutsch im Sprachvergleich - Grammatische Kontraste und Konvergenzen“ der Blick vom Deutschen ausgehend auf andere Sprachen gerichtet wird.

Jens Gerdes 